\title{
Should all men having a radical prostatectomy have a pelvic lymph node dissection? No
}

\author{
Firas Abdollah, MD; Maxine Sun, BSC; Rodolphe Thuret, MD; Pierre I. Karakiewicz, MD, FRCSC
}

Can Urol Assoc J 2010;4(6):425-6

$\mathrm{F}$ or decades, pelvic lymph node dissection (PLND) has been performed at the time of radical prostatectomy (RP) as a lymph node (LN) staging procedure in prostate cancer (PCa) patients. However, growing evidence suggests that PLND might not be an accurate tool for predicting lymph node invasion (LNI). Recently, Mattei and colleagues examined the primary prostate lymphatic drainage sites in PCa patients. " In their report, they defined "limited PLND" as the removal of LNs along the external iliac vein and obturator nerve; they reported that this dissection missed up to $62 \%$ of the primary LNs. Similarly, the extended PLND area, which also included LNs medial and lateral to the internal iliac vein, missed up to $37 \%$ of the primary LNs. The authors suggested a new "super extended" PLND area, which additionally includes the LNs along the common iliac vessels up to the ureteric crossing. However, the authors found that even this "super extended" PLND missed 25\% of the primary LNs. ${ }^{1}$ Consequently, the authors concluded that the removal of all primary LNs is not feasible in all or even most patients because of cost, time, extent of surgery and risk of complications. It is noteworthy that the complication rate after PLND may be up to $51 \%{ }^{2}$ the complication rate appears to be associated with increasing the extent of the dissection. ${ }^{3,4}$ These findings question the usefulness of PLND as a staging procedure in PCa patients. Moreover, these findings indicate the need to develop more accurate and, possibly less invasive, staging tools.

Even if we accept PLND as a LN staging procedure, its use in all patients is unjustifiable. Today, many preoperative staging tools may help to predict the presence of LN invasion. Nomograms represent the most commonly used tools in this field. ${ }^{5}$ Recent nomograms are up to $97.8 \%$ accurate. ${ }^{6,7}$ Most nomograms are based on simple and routinely available clinical and/or pathological predictors..$^{8-10}$ The advantages of these tools are their applicability to all patients regardless of their perioperative risk. Moreover, they do not contribute to additional operating time, additional cost and, most importantly, they do not contribute to morbidity. Since these tools can indicate in whom the PLND may be omitted, they may help to decrease the number of
PLNDs by limiting their indication to patients with high risk of LNI. For example, the National Comprehensive Cancer Network practice guidelines recommend PLND in patients with $\geq 2 \%$ risk of $\mathrm{LNI}$, according to the nomogram proposed by Cagiannos and colleagues..$^{10,11}$

Several studies could not find a link between PLND and cancer control outcomes. ${ }^{12-14}$ However, some authors suggested that PLND has a therapeutic benefit in PCa patients; ${ }^{15,16}$ they argue that this benefit may be touted as a recommendation to use PLND. Masterson and colleagues found that in patients with negative LNs, the removal of more LNs was associated with a more favourable biochemical recurrence-free rate (hazard ratio: $0.91, p=0.01$ )..$^{16}$ They speculated that this could be due to the removal of micrometastatic nodal disease. Similar results were reported by Joslyn and colleagues, who observed better cancer control outcomes when more LNs were removed. ${ }^{15}$ These results should be interpreted with caution. The retrospective nature of these studies indicates that the recorded benefit may be entirely due to reclassification bias, also known as the Will Rogers phenomenon. ${ }^{17}$ According to this theory, more extensive PLNDs, as opposed to limited PLNDs, result in more accurate staging and more accurately confirm the absence of nodal metastases. Accordingly, the more favourable cancer control outcomes in more extended PLNDs are not related to any form of therapeutic effect, but are attributed to a better staging.

The lack of randomized trials that address the effect of PLND (if any) on various cancer control outcomes preclude the possibility of a consensus on the value of PLND. However, even if we assume that PLND does have a beneficial cancer control effect, the very low LNI rate in contemporary patients may eliminate any meaningful benefit. Kawakami and colleagues ${ }^{18}$ examined the CaPSURE database; according to the $\mathrm{D}^{\prime}$ Amico risk classification, ${ }^{19}$ they reported $\mathrm{LNI}$ rates of $0.8,2.0$ and $7.1 \%$ for low, intermediate and high-risk PCa patients, respectively. Based on a $10 \%$ biochemical-free survival reported by Masterson and colleagues, ${ }^{16}$ the "number needed to treat" to achieve this beneficial effect in 1 individual is 1250, 500 and 140 PLNDs for low, intermediate, and high-risk patients, respectively. It may be hard to defend performing 1250, 500 or even 140 
Abdollah et al.

PLNDs to improve the biochemical recurrence-free rate by $10 \%$ in 1 individual. In that light, omitting the PLND appears to represent a much better option. The use of accurate preoperative non-invasive predictive tools, such as nomograms, should guide our medical decision and help us determine the patients who need PLND.

Cancer Prognostics and Health Outcomes Unit, University of Montreal Health Center, Montreal, Canada.

Competing interests: None declared.

This paper has been peer-reviewed.

\section{References}

1. Mattei A, Fuechsel FG, Bhatta Dhar N, et al. The template of the primary lymphatic landing sites of the prostate should be revisited: results of a multimodality mapping study. Eur Urol 2008:53:118-25.

2. Paul DB, Loening $S A$, Narayana $A S$, et al. Morbidity from pelvic lymphadenectomy in staging carcinoma of the prostate. J Urol 1983;129:1141-4.

3. Briganti A, Chun FK, Salonia A, et al. Complications and other surgical outcomes associated with extended pelvic lymphadenectomy in men with localized prostate cancer. Eur Urol 2006;50:1006-13

4. Stone NN, Stock RG, Unger P. Laparoscopic pelvic lymph node dissection for prostate cancer: comparison of the extended and modified techniques. J Urol 1997;158:1891-4.

5. Capitanio U, Jeldres C, Shariat SF, et al. Clinicians are most familiar with nomograms and rate their clinical usefulness highest, look-up tables are second best. Eur Urol 2008;54:958-9.

6. Karam JA, Svatek RS, Karakiewicz PI, et al. Use of preoperative plasma endoglin for prediction of lymph node metastasis in patients with clinically localized prostate cancer. Clin Cancer Res 2008;14:1418-22.

7. Briganti A, Gallina $\mathrm{A}$, Suardi $\mathrm{N}$, et al. A nomogram is more accurate than a regression tree in predicting lymph node invasion in prostate cancer. BJU Int 2008;101:556-60.
8. Briganti A, Chun FK-H, Salonia A, et al. Validation of a nomogram predicting the probability of lymph node invasion among patients undergoing radical prostatectomy and an extended pelvic lymphadenectomy. Eur Urol 2006; 49:1019-26; discussion 1026-7.

9. Briganti A, Karakiewicz PI, Chun FK, et al. Percentage of positive biopsy cores can improve the ability to predict lymph node invasion in patients undergoing radical prostatectomy and extended pelvic lymph node dissection. Eur Urol 2007:51:1573-81.

10. Cagiannos I, Karakiewicz P, Eastham JA, et al. A preoperative nomogram identifying decreased risk of positive pelvic lymph nodes in patients with prostate cancer. J Urol 2003;170:1798-803.

11. NCCN Clinical Practice Guidelines in Oncology. Prostate cancer. http://www.nccn.org/professionals/ physician_gls/PDF/prostate.pdf. Accessed November 10, 2010.

12. Berglund RK, Sadetsky N, Duchane J, et al. Limited pelvic lymph node dissection at the time of radical prostatectomy does not affect 5-year failure rates for low, intermediate and high risk prostate cancer: results from CaPSURE. J Urol 2007; 177:526-29; discussion 529-30.

13. DiMarco DS, Zincke H, Sebo TJ, et al. The extent of lymphadenectomy for pTXNO prostate cancer does not affect prostate cancer outcome in the prostate specific antigen era. J Urol 2005;173:1121-5.

14. Weight CJ, Reuther AM, Gunn PW, et al. Limited pelvic lymph node dissection does not improve biochemical relapse-free survival at 10 years after radical prostatectomy in patients with low-risk prostate cancer. Urology 2008;71:141-5.

15. Joslyn SA, Konety BR. Impact of extent of lymphadenectomy on survival after radical prostatectomy for prostate cancer. Urology 2006;68:121-5.

16. Masterson TA, Bianco FJ, Vickers AJ, et al. The association between total and positive lymph node counts, and disease progression in clinically localized prostate cancer. I Urol 2006;175:1320-4; discussion 1324-5.

17. Albertsen PC, Hanley JA, Barrows GH, et al. Prostate cancer and the Will Rogers phenomenon. J Natl Cancer Inst 2005;97:1248-53.

18. Kawakami J, Meng MV, Sadetsky N, et al. Changing patterns of pelvic lymphadenectomy for prostate cancer: results from CaPSURE. J Urol 2006;176(4 Pt 1):1382-6.

19. D'Amico AV, Whittington R, Malkowicz SB, et al. Biochemical outcome after radical prostatectomy, external beam radiation therapy, or interstitial radiation therapy for clinically localized prostate cancer. JAMA 1998;280:969-74.

Correspondence: Dr. Pierre Karakiewicz, Associate Professor and Director, Cancer Prognostics and Health Outcome Unit, University of Montreal Health Center (CHUM), 1058, rue St-Denis, Montreal, QC H2X 3J4; fax: 514-227-5103; pierre.karakiewicz@umontreal.ca or www.nomogram.org 